\title{
Extra Precautions While Caring Suspected COVID-19 Patient in ICU Beyond PPE and Hand Hygiene
}

\author{
Vitrag Shah ${ }^{1} \odot$, Niraj Tyagi ${ }^{2} \oplus$, Darshan Trivedi $^{3} \odot$
}

\begin{abstract}
Since the diagnosis of the first case of COVID-19 in December 2019, there have been reports of several healthcare workers infected with COVID19. It has changed the infection control practices of most ICUs all over the world. Prevention is better than cure is definitely proven true as there is no definite cure for COVID-19 yet. Personal protective equipment and hand hygiene are must while handling any suspected COVID-19 patient. Apart from that, there are several other things, which should be followed in ICU and specifically while caring for patient on ventilator. There are a large number of interventions done while treating any critically ill patient in ICU, which can generate aerosols and exaggerate spread of COVID-19, which include high-flow nasal cannula, NIV, nebulization, suctioning, bag and mask ventilation, endotracheal intubation, and bronchoscopy. We will be reviewing those things beyond PPE and hand hygiene along with the rationale of each of them, which can help to minimize the risk of exposure to healthcare workers and other patients in the surrounding. It will help not only to prevent COVID-19 transmission but also to reduce overall nosocomial infection rate. As per our knowledge, this will be the first paper reviewing innovative ideas to minimize the risk of infection in a comprehensive manner.

Keywords: Corona, COVID, COVID-19, High-flow nasal cannula (HFNC) oxygen therapy, ICU, Infection, Infection control, Mask, Nosocomial infection. Indian Journal of Critical Care Medicine (2021): 10.5005/jp-journals-10071-23767
\end{abstract}

\section{INTRODUCTION}

Since the diagnosis of the first case of COVID-19 in December 2019, there have been reports of several healthcare workers infected with COVID-19. It has changed the infection control practices of most ICUs all over the world. Prevention is better than cure is definitely proven true as there is no definite cure for COVID-19 yet. Personal protective equipment and hand hygiene are must while handling any suspected COVID-19 patient. Apart from that, there are several other things, which should be followed in ICU and specifically while caring for patient on ventilator. There are a large number of interventions done while treating any critically ill patient in ICU, which can generate aerosols and exaggerate spread of COVID-19, which include high-flow nasal cannula, NIV, nebulization, suctioning, bag and mask ventilation, endotracheal intubation, and bronchoscopy.

In this article, we will not be discussing routine infection control measures (e.g., proper PPE and mask/respirator usage, hand hygiene, isolation practices, disinfection of equipment, environmental cleaning, and spacing of beds) assuming they are already being followed. We will be reviewing various other minor but important things, which need to be considered for extra precautions, which can help to minimize the risk of exposure to healthcare workers and other patients in the surrounding (Fig. 8). It will help not only to prevent COVID-19 transmission but also to reduce overall nosocomial infection rate. As per our knowledge, this will be the first paper reviewing innovative ideas to minimize the risk of infection in a comprehensive manner.

1. Maximize air exchanges and negative pressure isolation ${ }^{1-3}$

- Either natural ventilation with airflow of at least $160 \mathrm{~L} / \mathrm{s}$ per patient or in negative pressure rooms with at least 12 \begin{tabular}{l}
\hline \hline 1,30partment of Critical Care Medicine, Kiran Multi Superspeciality \\
Hospital and Research Centre, Surat, Gujarat, India \\
${ }^{2}$ Department of Critical Care Medicine, Sir Ganga Ram Hospital, New \\
Delhi, Delhi, India \\
Corresponding Author: Vitrag Shah, Department of Critical Care \\
Medicine, Kiran Multi Superspeciality Hospital and Research Centre, \\
Surat, Gujarat, India, Phone: +91 9712909924, e-mail: dr.vitrag@gmail. \\
com \\
How to cite this article: Shah V, Tyagi N, Trivedi D. Extra Precautions \\
While Caring Suspected COVID-19 Patient in ICU Beyond PPE and Hand \\
Hygiene. Indian J Crit Care Med 2021;25(3):331-335. \\
Source of support: Nil \\
Conflict of interest: None \\
\hline \hline
\end{tabular}

air changes per hour is recommended during any aerosolgenerating procedures. HEPA filter unit is required for the recirculation of air. One hundred percent fresh air system is better but may not be available. Apart from this, the direction and pattern of airflow are also important, and it should be from clean to dirty and laminar distribution pattern. Use area with maximum number of air changes per hour to manage critically ill COVID-19 patient to minimize the risk of cross-infections. Most of the doctors may not be aware about heating, ventilation, and air conditioning (HVAC) system in their hospital. One should confirm the number of air changes with their maintenance department and intimate them to create negative pressure if possible. If negative pressure isolation is not available, ask maintenance staff if they can create relatively negative or less positive pressure by changing flow rates. 
2. Minimize transport and minimize opening and closing of the door ${ }^{4}$

- Whenever opening the door, close the door as soon as possible. Minimize transport of the patient inter- or intrahospital. Transport of patient may be required for several reasons, and it is really challenging to comply with full infection control measures. Proper planning of transport route and coordination between various departments are of upmost importance. Detailed discussion on transport protocol is beyond the scope of this article.

3. Oxygen

- Use minimum flow of oxygen required. Prefer low-flow oxygen delivery devices over high flow whenever possible. Use surgical/N95 mask over patient's face whenever using nasal cannula or any patient without artificial airway to minimize aerosol spread.

4. HME filter ${ }^{5-10}$

- Size of corona virus is $125 \mathrm{~nm} .{ }^{5}$ Due to such small size, filter is mandatory when managing any patient with COVID-19 on ventilator.

- Five types of devices are available:

o Heat and moisture exchanger (HME) without filter,

o Mechanical/hydrophobic filter,

o Electrostatic filter,

o Heat and moisture exchanger with mechanical/ hydrophobic filter (HMEF), and

o Heat and moisture exchanger with electrostatic filter (HMEF).

- Out of these five types, HME with electrostatic filter and HME without electrostatic filter are most commonly available and used in India.

- For bacterial and viral filters, one that filters particles in size $>0.2$ to $0.3 \mu \mathrm{m}$ with $>95 \%$ viral filtration efficiency (VFE) should be used. VFE of $99.99 \%$ means that only one particle in 104 will pass through the filter under standard test conditions and usually at flow rate of $30 \mathrm{~L} / \mathrm{min}$, so the higher the flow rate, the lower the efficiency.

- Mechanical filter has more dead space and requires minimum $150 \mathrm{ml}$ of tidal volume, but it is more efficient and less affected by water vapor.

- Use heat and moisture exchanger and high-efficiency bacterial/viral filter (HMEF) between catheter mount connected to facemask (when using NIV or doing bag and mask ventilation) or endotracheal or tracheostomy tube and Y-piece of ventilator circuit or Ambu bag/self-inflating bag (Fig. 1A).

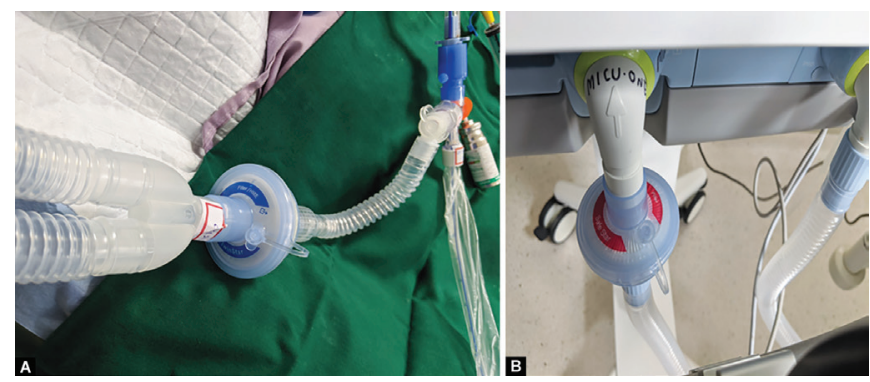

Fig. 1: (A) HME filter between catheter mount and Y-piece. (B) HME (without filter) at expiratory limb of ventilator circuit (right)
- Use high-efficiency bacterial/viral filter on the expiratory port of ventilator (Fig. 1B).

- Mechanical filters are preferred over electrostatic filters.

- Avoid using heated humidifier. Theoretically, it decreases the risk of room contamination during the disconnection of circuit but increases viral load in circuit. It increases the risk of blockage of the filter.

- Keep in mind higher resistance due to HME filter and change immediately when blocked or soiled. It should be changed routinely every 48 hours or as per manufacturer recommendation.

5. High-flow nasal cannula (HFNC) ${ }^{11,12}$

- HFNC is recommended by surviving sepsis campaign guidelines, and it is preferred over NIV whenever available.

- If using high-flow nasal cannula, use medical or N95 mask to cover patient's mouth and nose. Use minimum flow required to avoid aerosol spread.

6. Noninvasive ventilation (NIV) $)^{12}$

- Use full facemask over nasal or oronasal mask to minimize aerosol spread.

- Use mask with bronchoscopy port when doing bronchoscopy. Ideally, bronchoscopy should be avoided as it creates aerosol spread (Fig. 2).

- Mask should have tight seal and minimum leak.

- Mask should be nonvented. Vented masks should be avoided.

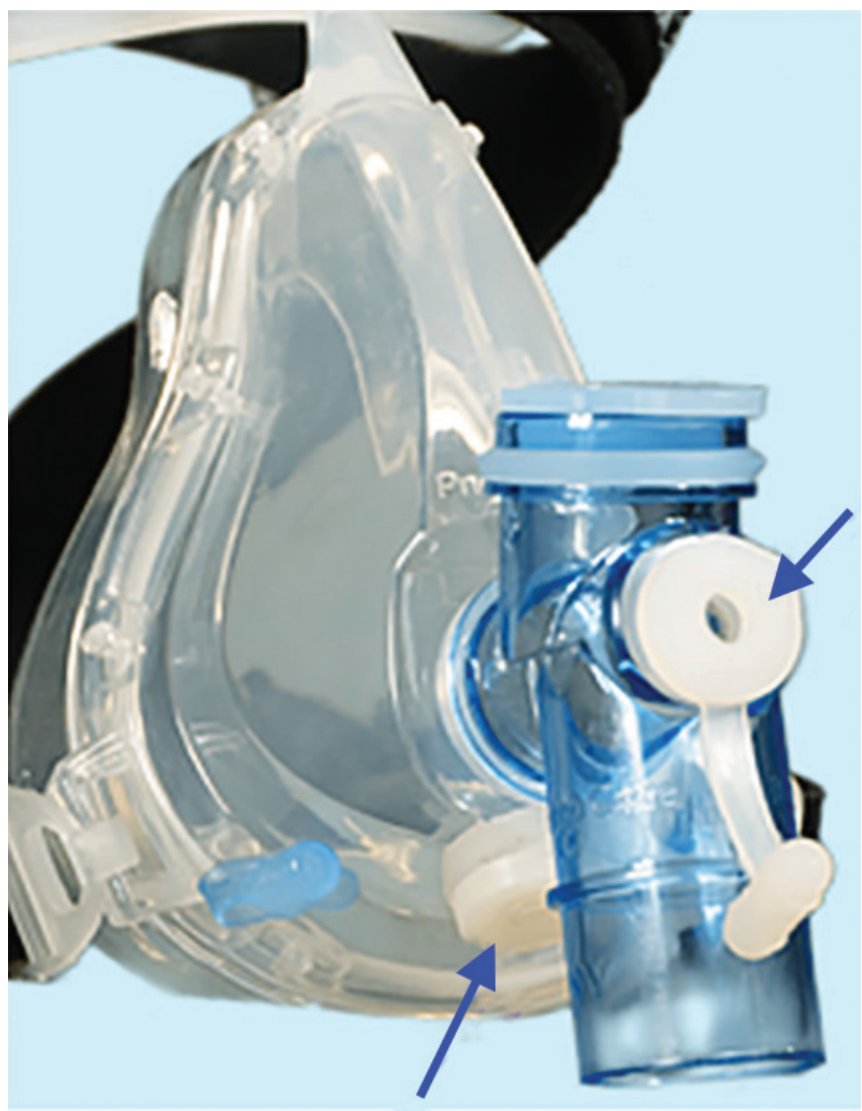

Fig. 2: NIV mask for bronchoscopy (upper arrow for oral route and lower arrow for nasal route) 
- Avoid using single-limb circuit over dual-limb circuit. If using portable CPAP machine and need to single-limb circuit, use exhalation valve in circuit and keep HME filter between facemask and exhalation valve in circuit.

- Use minimum effective pressure (pressure support/ inspiratory pressure and PEEP).

7. Precautions for intubation ${ }^{11,13-15}$

- Early versus late intubation is controversial as per available data till now. Decision to intubate should be individualized.

- It must be done by expert. Keep minimum staff in room. Totally, three individuals are required, which include one expert for intubation, one assistant, and one staff to give drugs.

- Rapid sequence intubation should be done.

- Keep low threshold for intubation to avoid crash intubation and minimize aerosol spread with HFNC/NIV.

- Avoid bag and mask ventilation.

- Preoxygenation for 3 to $5 \mathrm{~min}$.

- Mask ventilation through close circuit through ventilator rather than bag and mask ventilation if necessary.

- Use HME filter as discussed earlier whenever doing bag and mask ventilation or mask ventilation using ventilator.

- Supraglottic airway is preferred over facemask ventilation.

- Ensure complete neuromuscular blockage before attempting intubation and even before mask ventilation.

- Clamping ET tube/attach HME filter while intubation.

- Use video laryngoscope for intubation if available.

- Using plastic drapes or aerosol box during intubation. ${ }^{15}$ Role of aerosol box during intubation is controversial and not routinely recommended.

- Inflate cuff with air immediately after intubation to keep cuff pressure 25 to $30 \mathrm{~mm} \mathrm{Hg}$ and make sure there is no leak.

- Monitor cuff pressure every 6 to 8 hours and minimize leak.

- Higher risk of leak with peak inspiratory pressure higher than cuff pressure. Increase cuff pressure before performing any recruitment maneuver.

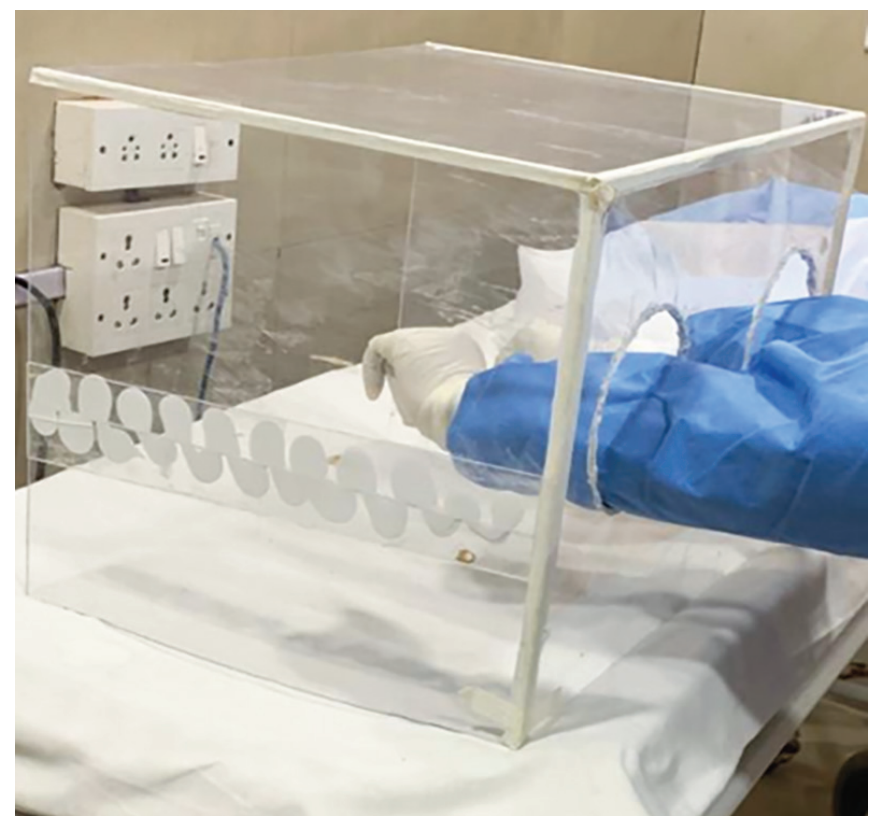

Fig. 3: Aerosol box for intubation and extubation
- Start mechanical ventilation only after inflating cuff to avoid leak and aerosolization.

8. Prepare for extubation

- Aerosol box and plastic drapes are also helpful and easier to use during extubation and then intubation ${ }^{15}$ (Fig. 3).

- Use of mild sedation (dexmedetomidine/fentanyl, etc.), intracuff lidocaine to avoid coughing during extubation ${ }^{13,20}$

- Start nasal oxygen/high-flow nasal cannula after extubation.

- Immediately apply surgical/N95 mask to face after extubation.

9. Precautions while disconnecting ventilator

- Minimize disconnection to avoid derecruitment and exposure of virus to environment. If disconnection is necessary (e.g., while shifting patient and emptying water in circuit), follow below-mentioned steps:

o Preoxygenation with $100 \%$ oxygen for 2 to $3 \mathrm{~min}$,

o Keep ventilator on standby,

o Clamp ET tube (if intubated),

o Disconnect (with direction of both disconnected ends facing down rather than up) circuit with HME still connected to patient, and

o Reconnect after doing activity for which it was disconnected and unclamp ET tube if clamped before reconnecting.

10. Cover equipment with transparent sheet ${ }^{8}$

- Covering ventilator with transparent plastic drape. Cover any other equipment (multipara monitor, X-ray, echo, USG machine, etc.) being used with plastic drape (Fig. 4). Placing the ventilator and syringe pump outside the room if feasible (e.g., through a wall port). This allows to do necessary settings and changes whenever required without exposure to staff, although no proven benefit.

11. Use disposable accessories 8

- Use disposable expiratory valve and new flow sensor when available. (Dragor has disposable expiratory valve available.) If not, clean and disinfect expiratory valve and flow sensor as per manufacturer instructions.

- If available, use disposable ECG leads, $\mathrm{SpO}_{2}$ probes, temperature probes, NIBP cuff, etc. Otherwise, clean and disinfect as per manufacturer recommendation before reuse.

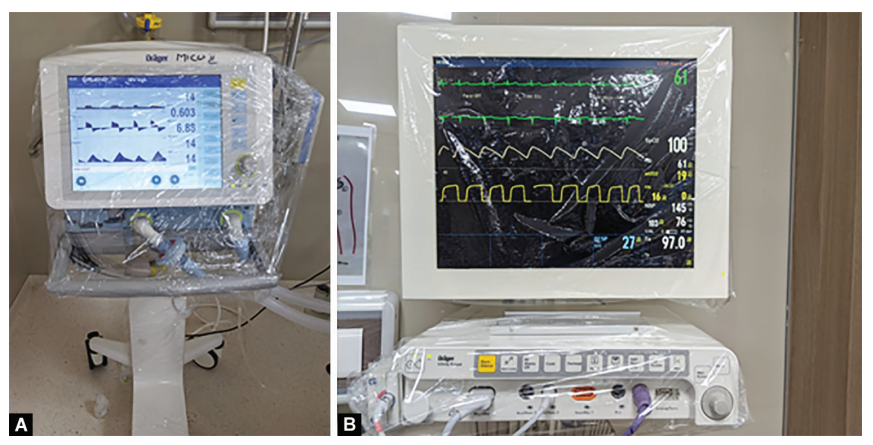

Fig. 4: Transparent plastic sheet covering (A) ventilator and (B) monitor 
12. Avoid routine suctioning ${ }^{16}$

- Use of close suction is mandatory to avoid aerosol spread when available.

- Continuous suctioning can be done during aerosolgenerating procedures like intubation, tracheostomy, and bronchoscopy as it helps to minimize the risk of nosocomial transmission.

13. Minimize nebulization ${ }^{12,17}$

- Nebulization creates aerosolization, so unnecessary routine nebulization should be avoided.

- MDI with spacer is preferred if nebulization required.

- MDI can be used along with close suction device as well, which has separate port/adapter for MDI (Fig. 5).

- If cannot use MDI and nebulization is essential, use jet nebulizer or vibrating mesh nebulizer, which should be inline close circuit nebulization from ventilator.

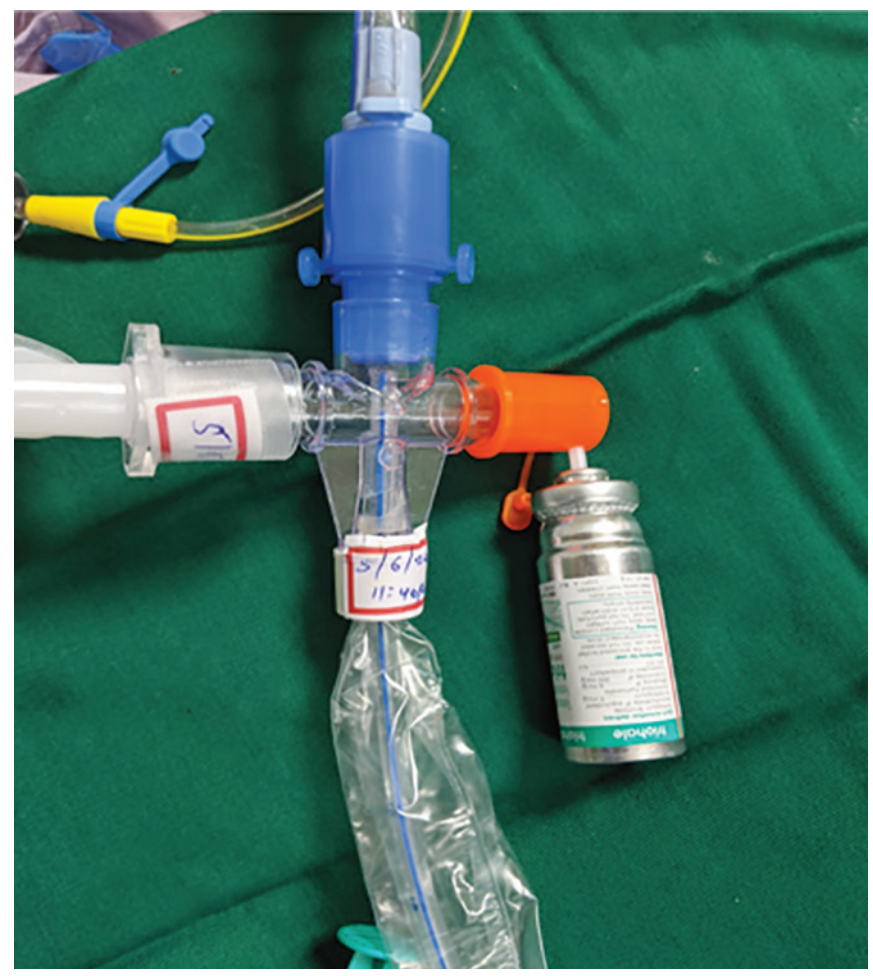

Fig. 5: Close suction with MDI attached to MDI adapter

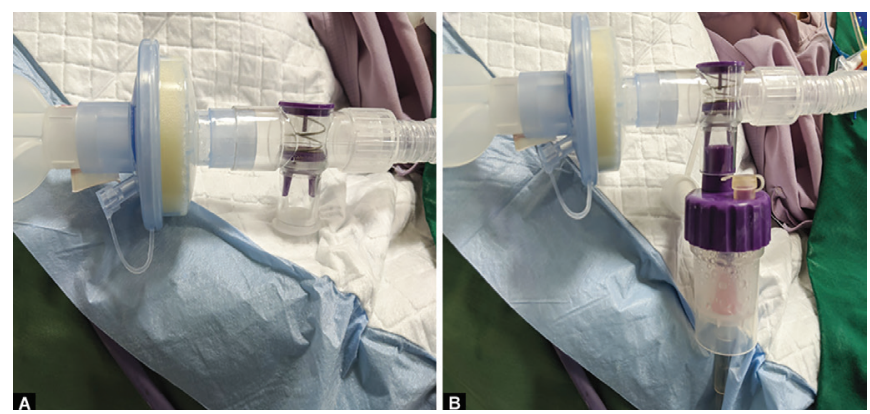

Fig. 6: Novel T-connector for nebulization with auto shut-off function. Without nebulizer chamber (left), nebulizer chamber attached to T-connector (right)
- Whenever available, use T-connector with auto shutoff function (one-way valve) to attach nebulizer chamber and use HME filter with switch to on or off (bypass) HME filter to avoid disconnection and keep circuit closed before and after nebulization (Figs 6 and 7).

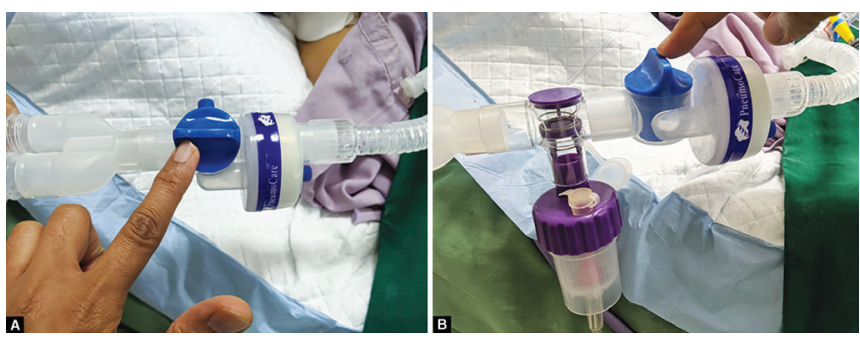

Fig. 7: Novel HME design. HME active (left) and HME bypassed during nebulization (right)

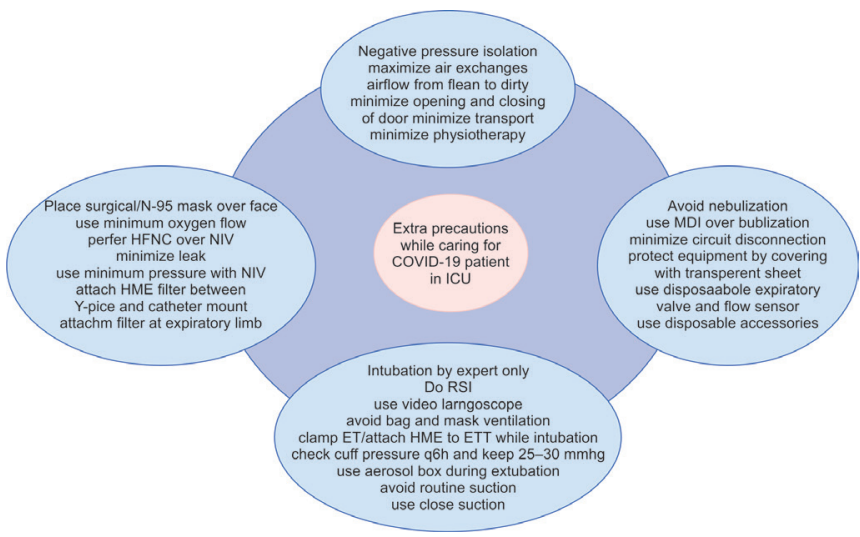

Fig. 8: Overview of extra precautions while caring for COVID-19 patient in ICU

14. Minimize chest physiotherapy ${ }^{18,19}$

- Most patients with COVID-19 do not require routine chest physiotherapy. Most interventions in respiratory physiotherapy are potentially aerosol generating, so it should be done only when deemed necessary. Italian association of respiratory physiotherapist and international experts in cardiorespiratory physiotherapy have come up with recommendation for physiotherapy in the management of COVID-19 patients. Each hospital should make their own policy and protocol as per available resources and feasibility, and standard preventive measures must be followed.

- Apart from the PPE kit, hand hygiene, and above measures, there are some in vitro studies of povidone-iodine nasal solution to mitigate virus transmission. It has been found to be effective for contact time as short as 15 seconds and even in $0.5 \%$ concentration, but further clinical studies are needed to establish benefit. ${ }^{21}$

\section{OrCIDS}

Vitrag Shah $\odot$ https://orcid.org/0000-0001-8337-7708

Niraj Tyagi (1) https://orcid.org/0000-0001-5862-9731

Darshan Trivedi $\odot$ https://orcid.org/0000-0002-4186-0854 


\section{References}

1. World Health Organization. Infection prevention and control during health care when COVID-19 is suspected: interim guidance. Geneva: World Health Organization; 2020.

2. Rungta N, Zirpe KG, Dixit SB, Mehta Y, Chaudhry D, Govil D, et al. Indian Society of Critical Care Medicine Experts Committee Consensus Statement on ICU planning and designing, 2020. Indian J Crit Care Med 2020;24(Suppl 1):S00. DOI: 10.5005/jp-journals-10071-G23185.

3. Saran S, Gurjar M, Baronia A, Sivapurapu V, Ghosh PS, Raju GM, et al. Heating, ventilation and air conditioning (HVAC) in intensive care unit. Crit Care 2020;24:194. DOI: 10.1186/s13054-020-02907-5.

4. Liew MF, Siow WT, Yau YW, See KC. Safe patient transport for COVID19. Crit Care 2020;24(1):94. DOI: 10.1186/s13054-020-2828-4.

5. Fehr AR, Perlman S. Coronaviruses: an overview of their replication and pathogenesis. In: Coronaviruses. New York: Humana Press; 2015, pp. 1-23.

6. Dragor. Guideline breathing system filter. April 2020.

7. Dragor. Filter/HMEs: supporting daily clinical routine.

8. Dragor. SARS-CoV-2 and handling of medical devices for intensive care (January 28, 2020) updated March 24, 2020.

9. Wilkes AR. Heat and moisture exchangers and breathing system filters: their use in anaesthesia and intensive care. Part 1 - history, principles and efficiency. Anaesthesia 2011;66(1):31-39. DOI: 10.1111/j.1365-2044.2010.06563.x.

10. Wilkes AR. Heat and moisture exchangers and breathing system filters: their use in anaesthesia and intensive care. Part 2 practical use, including problems, and their use with paediatric patients. Anaesthesia 2011;66(1):40-51. DOI: 10.1111/j.13652044.2010.06564.x.

11. Alhazzani W, Møller MH, Arabi YM, Loeb M, Gong MN, Fan E, et al. Surviving sepsis campaign: guidelines on the management of critically ill adults with coronavirus disease 2019 (COVID-19). Intensive Care Med 2020;46(5):854-887. DOI: 10.1007/s00134-020-06022-5.

12. Respiratory care Committee of Chinese Thoracic Society. Expert consensus on respiratory therapy related to new coronavirus infection in severe and critical patients. Zhonghua Jie $\mathrm{He} \mathrm{He} \mathrm{Hu}$ Xi Za Zhi 2020;17:E020. DOI: 10.3760/cma.j.issn.1001-0939.2020. 0020.
13. Cook, TM, El-Boghdadly K, McGuire B, McNarry AF, Patel A, Higgs A. Consensus guidelines for managing the airway in patients with COVID-19: guidelines from the Difficult Airway Society, the Association of Anaesthetists the Intensive Care Society, the Faculty of Intensive Care Medicine and the Royal College of Anaesthetists. Anaesthesia 2020;75(6):785-799. DOI: 10.1111/anae.15054.

14. Apeksh P, Shah A, Garg R, Divatia JV, Kundra P, Doctor JR, et al. All India difficult airway association (AIDAA) consensus guidelines for airway management in the operating room during the COVID-19 pandemic. Indian J Anaesth 2020;64(14):107. DOI: 10.4103/ija.IJA_498_20.

15. Matava CT, Yu J, Denning S. Clear plastic drapes may be effective at limiting aerosolization and droplet spray during extubation: implications for COVID-19. Can J Anaesth 2020;67(7):902-904. DOI: 10.1007/s12630-020-01649-w.

16. Chan, MTV, Chow BK, Lo T, Ko FW, Ng SS, Gin T, et al. Exhaled air dispersion during bag-mask ventilation and sputum suctioningImplications for infection control. Sci Rep 2018;8(1):198. DOI: 10.1038/ s41598-017-18614-1.

17. Maves RC, Mukherjee V. Management of patients with COVID-19 infection. Chestnet.Org. https://www.chestnet.org/Guidelines-andResources/Resources/Management-of-Patients-with-COVID-19Infection.

18. Thomas P, Baldwin C, Bissett B, Boden I, Gosselink R, Granger CL, et al. Physiotherapy management for COVID-19 in the acute hospital setting: clinical practice recommendations. J Physiother 2020; 66(2):73-82. DOI: 10.1016/j.jphys.2020.03.011.

19. Lazzeri M, Lanza A, Bellini R, Bellofiore A, Cecchetto S, Colombo A, et al. Respiratory physiotherapy in patients with COVID-19 infection in acute setting: a Position Paper of the Italian Association of Respiratory Physiotherapists (ARIR). Monaldi Arch Chest Dis 2020;90(1):1285. DOI: 10.4081/monaldi.2020.1285

20. Peng $F$, Wang $M$, Yang $H$, Yang $X$, Long $M$. Efficacy of intracuff lidocaine in reducing coughing on tube: a systematic review and meta-analysis. J Int Med Res 2020;48(2):300060520901872. DOI: $10.1177 / 0300060520901872$

21. Frank S, Brown SM, Capriotti JA, Westover JB, Pelletier JS, Tessema $B$. In vitro efficacy of a povidone-iodine nasal antiseptic for rapid inactivation of SARS-CoV-2. JAMA Otolaryngol Head Neck Surg 2020;146(11):1-5. DOI: 10.1001/jamaoto.2020.3053. 